\title{
Fuzzy Dependencies between Preparedness and Learning Outcome
}

\author{
S. Encheva ${ }^{1}$, S. Tumin ${ }^{2}$ \\ ${ }^{1}$ Stord/Haugesund University College \\ Bjørnsonsg. 45, 5528 Haugesund \\ Norway, sbe@hsh.no \\ ${ }^{2}$ IT Dept., University of Bergen \\ PO Box 7800, 5020 Bergen \\ Norway, edpst@it.uib.no
}

\begin{abstract}
There is a large number of learning management systems as well as intelligent tutoring systems supporting today's educational process. Some of these systems relay heavily on use and reuse of learning objects. A lot of work has been done on creating, storing, classifying and filtering learning objects with respect to a specific subject. Considerable amount of research focuses on facilitating the process of reusing already available learning objects. This work is devoted to a study of a decision making process related to recommending the most appropriate learning objects to each particular student.
\end{abstract}

\section{Introduction}

Learning objects are the core concept in an approach to learning content in which content is broken down into "bite size" chunks. These chunks can be reused, independently created and maintained, and pulled apart and stuck together like so many legos, [25].

Learning technology systems and interoperability standards providing reuse of learning objects and interoperability of content across delivery are developed by [21], [22], and [23].

SCORM [22] provides technical standards that enable web-based learning systems to find, import, share, reuse, and export learning content in a standardized way. However, SCORM is written for toolmakers who know what they need to do to their products to conform with SCORM technically.

Please use the following format when citing this chapter: 
IEEE Learning Object Metadata [21] defines a set of resource description framework constructs that facilitates introduction of educational metadata into the semantic web.

HarvestRoad Hive [23] is an independent, federated digital repository system. It enables the collection, management, discovery, sharing and reuse of LOs used in the delivery of online courses within higher education.

A lot of work has been done on creating, storing, classifying and filtering learning objects with respect to a specific subject. Considerable amount of research focuses on facilitating the process of reusing already available learning objects. This work is devoted to a study of a decision making process related to recommending the most appropriate learning objects to a particular student.

\section{The Model}

\subsection{Conceptual modeling}

A level-based instruction model is proposed in [11]. A model for student knowledge diagnosis through adaptive testing is presented in [3]. An approach for integrating intelligent agents, user models, and automatic content categorization in a virtual environment is presented in [6].

A learning style is the general, habitual mode of processing information; it is a predisposition on the part of some students to adopt a particular learning strategy regardless of the specific demands of the learning task: that is, individuals' learning styles are simply the cognitive styles that they evidence when confronted with a learning task [15]. According to the Kolb's model [8] there are four learners types - concrete, reflective, abstract, reflective, abstract, active, and concrete, active.

The three learning preferences are auditory (learning by hearing), visual (learning by seeing), and kinesthetic (learning by doing), [2].

Student learning orientations [9] are critical for individualizing the instructional process. The four learning orientations are transforming learners, performing learners, conforming learners, and resistant learners.

\subsection{Concept lattices}

A concept is considered by its extent and its intent: the extent consists of all objects belonging to the concept while the intent is the collection of all attributes shared by the objects [19]. A context is a triple $(G, M, I)$ where $G$ and $M$ are sets and $I \subset G \times M$. The elements of $G$ and $M$ are called objects and attributes respec- 
tively. The set of all concepts of the context $(G, M, I)$ is a complete lattice and it is known as the concept lattice of the context $(G, M, I)$.

\subsection{Fuzzy membership}

Fuzzy reasoning methods [20] are often applied in intelligent systems, decision making and fuzzy control.

A prediction method in [4] applies formal concept analysis and fuzzy inference. In particular it shows how to calculate the value of a membership function of an object if the object belongs to a particular concept. The sum-of-1-criterion states that $\Sigma_{i \in M i} \mu_{i}(x)=1, \forall x \in \chi$, where $M_{i}, i=1, \ldots, k$ denotes all possible membership terms $\left\{m_{i}, i=1, \ldots, k\right\}$ of a fuzzy variable in some universe of discourse $\chi$.

An affiliation value to a concept represents the relative extent to which an object belongs to this concept or an attribute is common to all objects in the concept. The threshold for membership values is regarded as significant. This is obtained by computing the arithmetic mean of all entries within a column and take it as a threshold.

\subsection{Multi criteria decision making methods}

An extensive bibliography review on multi criteria decision making methods are summarised in [5] and [16]. Further interesting discussions may be found in [1], [7], [10] and [12].

Most human beings highly appreciate assistance in a form of well structured technique while working with complex decisions. The Analytic Hierarchy Process (AHP) developed by T. Saaty in 1980 [13] is one of those techniques and it has been applied in a number of different areas like government, business, industry, healthcare, and education. AHP involves mathematical programming, quality function deployment, meta-heuristics, strengths, weaknesses, opportunities and threats analysis and data envelopment analysis, [17] and [18]. The underlying concepts of AHP are structuring the complex decision problem as a hierarchy of goal, criteria and alternatives, pair-wise comparison of elements at each level of the hierarchy with respect to each criterion on the preceding level, and finally vertically synthesizing the judgments over the different levels of the hierarchy.

The analytic hierarchy process is a multi criteria decision making method [14] for working with multi attribute problems. A complex problem is broken down into smaller parts, further organized into levels. Then a hierarchical structure is generated. The goal is to determine the impact of a lower level on an upper level by paired comparisons done by a decision maker.

AHP facilitates estimation of the impact of each alternative on the overall objective of the hierarchy it is used as a consistency test to filter out inconsistent judgements. 


\subsection{The scenario}

In this scenario all students, within a particular subject, are suggested to take a web based test at the beginning of a semester. Test results indicate lack of knowledge and skills, lack of understanding of certain concepts or misconceptions, that are prerequisites for studying that subject. Based on the test results students are placed in different groups. Suitable learning objects are later on suggested to each student based on her group membership.

At the initial stage group types are formed based on previous teaching experience. If such experience is missing the groups can be formed according to a lecture assumption. Group types are further tuned when more experience is obtained.

The theory of concept lattices is applied in establishing relationships among groups of learners and the subject content. The process of assigning a student to a particular group is based on fuzzy functions. Such functions allow partial group membership, i.e. a particular individual may belong to some extend to more than one group. This in contrast to classical set theory where an element is either within a set or does not belong to that set, [24]. This makes the approach much more dynamic, flexible and easy to adapt to the individual needs of each student.

Learning objects are first collected in a database. Metadata is attached to each learning object, describing content, size, purpose and recommended educational level. AHP methods are applied for assigning a learning object to a group and consequently to a student.

\section{System}

The system prototype is build as a Web-based application using Apache HTTP server, mod_python module and SQLite database. The mod_python module provides programmable runtime support to the HTTP server using Python programming language. The whole application components:

- Web-based users interface,

- application logic, and

- database interaction were written in Python.

The system implementation has Web application server architecture:

the presentation layer is handled by an Apache Web server,

the logic layer is written in Python, and

the data layer is implemented using SQLite database engine.

Python provides a programming environment for implementing script-based handler for dynamic content, data integration and users' software agents. The back end SQLite databases are used to store both static and dynamic data. Apache is a modular Web server that can incorporate a high level scripting language as a module such as f. ex. mod_python. Using mod_python, python interpreter becomes a part of the Web server. SQLite is a small footprint, zero-administration 
and serverless databasesystem. SQLite stores persistence data into files. SQLite thus provides a database platform for multiple databases.

\section{Conclusion}

While most efforts aim at providing a technology to access and share existing learning objects, much less is known about how to assign the most suitable learning objects for a student.

The proposed method can be used to determine the learning effect of using learning objects in a subject as well as qualities of a single learning object. Learning styles and learning preferences can be further employed in the process of choosing the most appropriate learning object for each student. In addition the applications of fuzzy functions allow partial group membership, i.e. a particular individual may belong to several groups. This makes the approach much more dynamic, flexible and easy to adapt to the individual needs of each student.

\section{References}

1. B. Cillo and T. Saaty, The Encyclicon, Volume 2: A Dictionary of Complex Decisions using the Analytic Network Process, 2008

2. R.Dunn, K.Dunn and G.Price, Manual: Learning style inventory, Lawrence, KS: Price Systems, 1985

3. E. Guzman, and R. Conejo, A model for student knowledge diagnosis through adaptive testing, Lecture Notes in Computer Science, vol. 3220, Springer-Verlag, Berlin Heidelberg New York, 2004, pp. 12-21

4. C. S. Herrmann, S. Holldobler, A. Strohmaier, Fuzzy conceptual knowledge processing, ACM Symposium on Applied Computing, 1996, pp. 628-632

5. W. Ho, Integrated analytic hierarchy process and its applications - a literature review. European Journal of Operational Research, vol. 186(1), pp. 211-228

6. D. Huffman, F. Goldberg, and M. Michlin, Using computers to create constructivist environments: impact on pedagogy and achievement, Journal of Computers in Mathematics and Science Teaching, vol. 22(2), 2003, pp. 151-168

7. K. P. Kearns and T. Saaty, Analytical Planning: The Organization of Systems, Pergamon, 1985

8. D. A. Kolb, Experiential Learning: Experience as the Source of Learning and Development, Englewood Cliffs, NJ: Prentice-Hall, 1984

9. M. Martinez and C. V. Bunderson, Building interactive Web learning environments to match and support individual learning differences, Journal of Interactive Learning Research, vol. 11(2), 2000, pp. 163-195.

10. M. S. Özdemir and T. Saaty, The Encyclicon, A Dictionary of Decisions with Dependence and Feedback based on the Analytic Network Process, with Müjgan S. Özdemir, 2005

11. C. Park, and M. Kim, Development of a Level-Based Instruction Model in Web-Based Education, Lecture Notes in Artificial Intelligence, vol. 3190, Springer-Verlag, Berlin Heidelberg New York, 2003, pp. 215-221 
12. K. Peniwati and T. Saaty, Group Decision Making: Drawing Out and Reconciling Differences, 2007

13. T. Saaty, The Analytic Hierarchy Process: Planning, Priority Setting, Resource Allocation, McGraw-Hill, 1980

14. T. Saaty, Theory and Applications of the Analytic Network Process: Decision Making with Benefits, Opportunities, Costs and Risks, ISBN 1-888603-06-2, 2005

15. R.Schmeck, Learning Strategies and Learning Styles. New York: Plenum Press, 1988

16. R. E. Steuer, and P. Na, Multiple Criteria Decision Making Combined with Finance: A Categorized Bibliography, European Journal of Operational Research, vol. 150(3), 2003, pp. 496-515.

17. O.S.Vaidya and S.Kumar, Analytic hierarchy process: An overview of applications. European Journal of Operational Research, vol. 169(1), February 2006, pp. 1-29.

18. L.G. Vargas and T.Saaty, Decision Making with the Analytic Network Process: Economic, Political, Social and Technological Applications with Benefits, Opportunities, Costs and Risks, Springer, 2006

19. R. Wille, Concept lattices and conceptual knowledge systems, Computers Math. Applications, vol. 23(6-9), 1992, pp. 493-515

20. L.A.Zadeh, The concept of linguistic variable and its applications to approximate reasoning, Parts I, II, III, Information Sciences, 8(1975) pp. 199-251; 8(1975) pp. 301-357; 9(1975) pp. 43-80.

21. http://www.harvestroad.com/

22. http://www.adlnet.org/index.cfm?fuseaction=scormabt

23. http://kmr.nada.kth.se/el/ims/md-lomrdf.html

24. http://www.engsc.ac.uk/journal/index.php/ee/article/ view/

25. http://www.eduworks.com/LOTT/Tutorial/ 